\section{H.I. Ali}

Control and Systems

Engineering Department,

University of Technology

Baghdad, Iraq

60143@uotechnology.edu.iq

\section{H-infinity Model Reference Controller Design for Magnetic Levitation System}

Abstract- In this paper, a new robust approach based on combining the model reference control with $H$-infinity technique is proposed. The goal of the proposed controller is to obtain an adequate transient response which may not be obtained when only H-infinity technique is used. This is done by adding the model reference block to the standard $H$-infinity feedback configuration. Then the overall block diagram is formulated by linear fractional transformation (LFT). The Magnetic Levitation which is a highly nonlinear, open loop unstable and uncertain system is used to show the effectiveness of the proposed controller. The results show that the proposed controller is very effective in compensating the system parameters variations with forcing the system output to track the output of the model reference. A variation of $\pm 10 \%$ in system parameters is considered.

Keywords- Robust control, H-infinity control, Model reference control, Magnetic levitation system.
Received on: 30/11/2016 Accepted on: 20/12/2017

How to cite this article: H.I. Ali, "H-infinity Model Reference Controller Design for Magnetic Levitation System," Engineering and Technology Journal, Vol. 36, Part A, No. 1, pp. 17-26, 2018.

\section{Introduction}

The magnetic levitation system has gained a big interest in the entire world because of its great practical importance in engineering fields. They are characterized as unstable open loop, highly nonlinear and uncertain systems. Furthermore, the magnetic levitation systems have high steady state error and with a small change in system parameters, they may be destabilized. Therefore, the modeling and control are very challenging for such as systems. On the other hand, the magnetic levitation technology helps in eliminating friction losses due to mechanical contact. Some of applications of magnetic levitation systems include magnetic bearings, high-speed trains and high precision platforms [1,2].

Many approaches have been applied to control the magnetic levitation system, including type 2 fuzzy logic controller [3], sliding mode control combined with fuzzy logic control [4] and optimal robust control $[5,6]$.

Santosh [1] presented the design of robust feedback control for magnetic levitation system. The $\mathrm{H}$ infinity control was investigated to stabilize the magnetic levitation system by keeping a magnetic ball suspended in the air. However, there were no specific transient response specifications to be achieved. However, in all previous literatures there was no specific time response specifications required to be achieved.

On the other hand, the H-infinity optimal control represents one of the powerful approaches in robust control system design. Although the $\mathrm{H}$-infinity control is an effective method in rejecting the disturbance and noise control systems, the robust design approach may not obtain an adequate transient response specification [7]. On the other hand, to achieve an asymptotic tracking of prescribed limits, a suitable model reference can be considered and its output is used as the desired plant response [8].

In this work a combination of $\mathrm{H}$-infinity and model reference control is proposed for magnetic levitation system. The main objective of this combination is to synthesize a feedback controller that achieves an asymptotic tracking of prescribed reference output while compensating the system uncertainty and rejecting the disturbances. The controller is adjusted such that the system output tracks as close as possible the output of a given model reference.

\section{System Mathematical Model}

The ferromagnetic ball suspended in a voltagecontrolled magnetic field represents the main components of the magnetic levitation system. The schematic diagram of the magnetic levitation system can be shown in Figure 1. The electromagnetic units play an important role in magnetic levitation control in transferring the measured signals to the PC by input/output card. The control signals will be transferred from the PC to magnetic levitation and back via the analogue control interface. The mechanical and electrical units of the system are presented in Figure 2 [9]. On the other hand, the magnetic levitation system components are: electromagnet, a metal sphere and an infra-red sphere position sensor. The magnetic ball suspension system is divided into two parts: mechanical part and electromagnetic part. The 
mechanical part of the magnetic levitation system is considered at first. Figure 3 shows the free body diagram of the system when the ball is suspended due to balancing the electromagnetic force $f_{e m}$ and the force due to gravity $f_{g}$ [9]. Newton's 3rd law of motion can be used for representing the net force $f_{n e t}$ acting on the ball with neglecting the friction. the drag force of the air is given by $[1,10]$ :

$f_{n e t}=f_{g}-f_{e m}$

$m \ddot{x}=m g-f_{e m}$

where $f_{e m}=c \frac{i^{2}}{x^{2}}$ and $c$ is a constant depending on the coil (electromagnet) parameters, $i$ is the coil current of electromagnet and $x$ is the position of the ball. $f_{\text {em }}$ denotes the magnetic force generated by the coil. $m$ is the metal sphere mass and $g$ is the gravitational force. At equilibrium state the magnetic will equal to the force of gravity on the object and the object acceleration will equal to zero. Consequently, equation (2) will be [2]:

$m g=c \frac{i_{0}^{2}}{x_{0}^{2}}$

where $i_{0}$ represents the current coil of electromagnet at equilibrium point, $x_{o}$ is the ball position at equilibrium point.

The magnetic ball position will be influenced by the inductance of the electromagnet coil. The point between the electromagnetic force and gravity (levitating force) is inherently unstable. To solve this problem, the nonlinear electromagnetic force can be linearized. At equilibrium state, the following equation is obtained $[1,2]$ :

$m \ddot{x}=c\left(\frac{2 i_{0}^{2}}{x_{0}^{3}}\right) x-c\left(\frac{2 i_{0}}{x_{0}^{2}}\right) i$

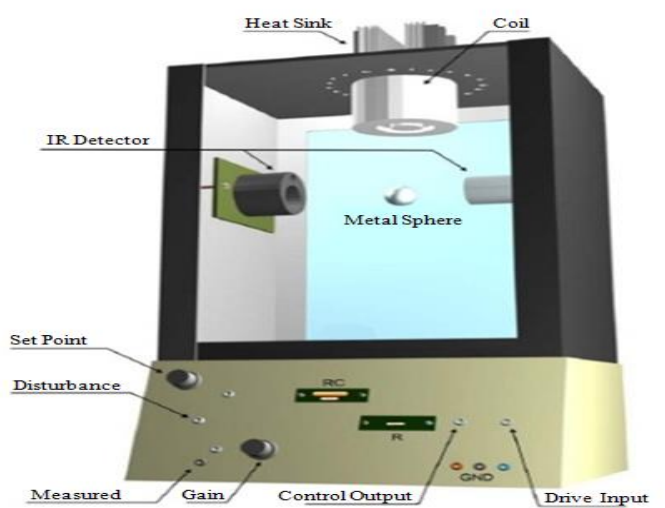

Figure 1: Electromagnetic levitation system [9]

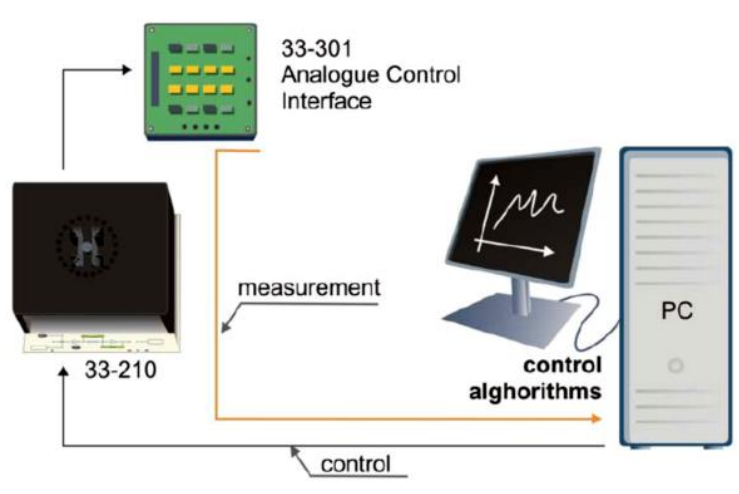

Figure 2: Magnetic levitation control system [9]

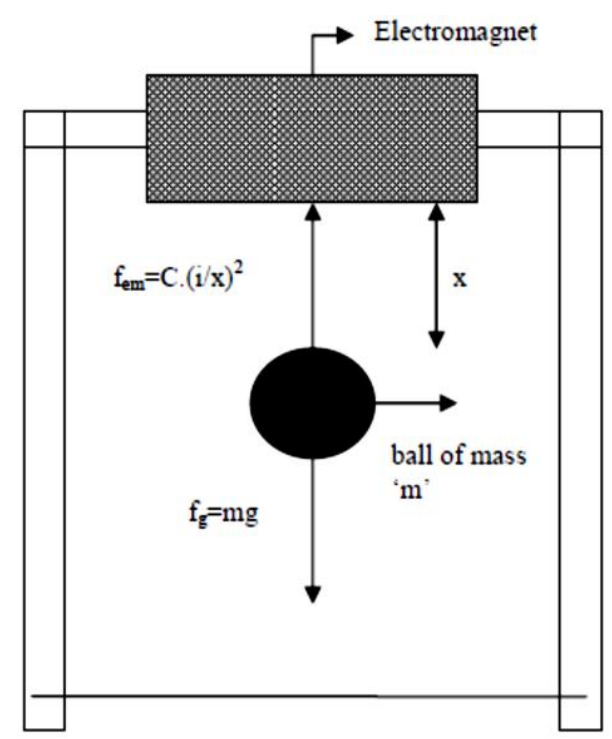

Figure 3: Free Body diagram of magnetic levitation system [9] 
The electromagnetic part of the system is shown in Figure 4. By applying Kirchhoff's voltage law as:

$e_{\text {in }}-i_{R} R=L \frac{d i_{L}}{d t}$

Then by using equations (4) and (5), the total magnetic levitation system model is expressed by: $\left[\begin{array}{l}\dot{x}_{1} \\ \dot{x}_{2} \\ \dot{x}_{3}\end{array}\right]=\left[\begin{array}{ccc}\frac{-R}{L} & 0 & 0 \\ \frac{-k_{i}}{m} & 0 & \frac{k_{S}}{m} \\ 0 & 1 & 0\end{array}\right]\left[\begin{array}{l}x_{1} \\ x_{2} \\ x_{3}\end{array}\right]+\left[\begin{array}{c}\frac{1}{L} \\ 0 \\ 0\end{array}\right] E_{\text {in }}$ $y=\left[\begin{array}{lll}0 & 0 & 1\end{array}\right]\left[\begin{array}{l}x_{1} \\ x_{2} \\ x_{3}\end{array}\right]$

where $\quad k_{s}=c\left(\frac{i_{0}^{2}}{x_{0}^{3}}\right), k_{i}=c\left(\frac{2 i_{0}}{x_{0}^{2}}\right), x_{1}(t)$ represents the position of the metal sphere ball, $x_{2}(t)$ represents the velocity of the metal sphere ball and $x_{3}(t)$ represents the acceleration of the metal sphere ball. The values of the parameters of the system are listed in Table 1.

\section{Controller Design}

The transformation into standard configuration can be done using Lower Fractional Transformation (LFT) technique. LFT is a technique which specify grouping signals into sets of external inputs ( $w$ ) and outputs $(z)$, input to the controller $(v)$ and output from the controller (control signal $u$ ). The $\mathrm{H}$-infinity controller design depends on minimizing the infinity norm from input $w=\left[\begin{array}{l}r \\ d\end{array}\right]$ (set point $r$, disturbance $d$ ) to output $z=\left[\begin{array}{l}e_{p} \\ e_{u}\end{array}\right]$ where $e_{p}$ represents the weighted error signal and $e_{u}$ represents the weighted control signal. The standard H-infinity control problem is shown in Figure 5. Where $K(s)$ represents the desired optimal controller, $P(s)$ represents the plant transfer matrix and it is known as augmented plant [11]. Normally, the augmented plant includes the plant model in addition to the weighting functions that reflect the design specifications. In this work, we proposed to add the transfer function of the model reference to the augmented plant $P(s)$. The error between the two outputs (system and model reference) is selected to be one of the outputs to be minimized as shown in Figure 6 . The performance weighting function is used to weight the error between the outputs of the system and the model reference $\left(e_{r}\right)$ to keep the system output within prescribed limits represented by model reference.

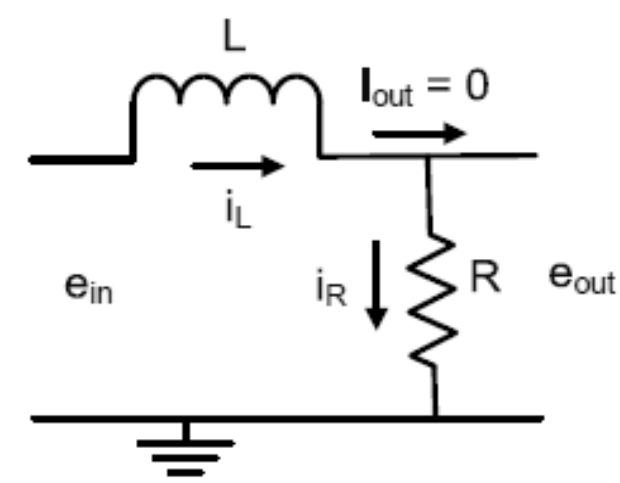

Figure 4: Magnetic levitation system (electromagnetic part)

Table 1: List of system parameters [1]

\begin{tabular}{cc}
\hline Parameter & Nominal value \\
\hline$R$ & $10 \Omega$ \\
$L$ & $0.4125 \mathrm{H}$ \\
$m$ & $0.068 \mathrm{~kg}$ \\
$g$ & $9.81 \mathrm{~m} / \mathrm{s}^{2}$ \\
$i_{o}$ & $0.8 \mathrm{Amp}$ \\
$x_{o}$ & $0.012 \mathrm{~m}$ \\
$c$ & $6.53 \times 10^{-5}$ \\
\hline
\end{tabular}

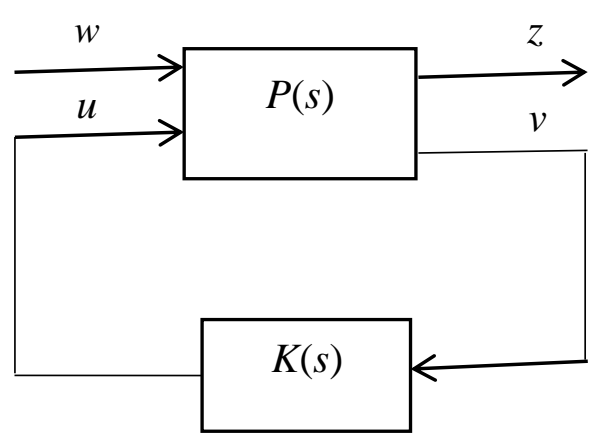

Figure 5: Standard H-infinity control problem [11]

The dynamics of the system shown in figure 5 can be expressed as [11]:

$$
\begin{aligned}
& \dot{x}=A x+B_{1} w+B_{2} u \\
& z=C_{1} x+D_{11} w+D_{12} u \\
& y=C_{2} x+D_{21} w+D_{22} u
\end{aligned}
$$

Then the plant transfer matrix can be partitioned as follows $[11,12]$ : 


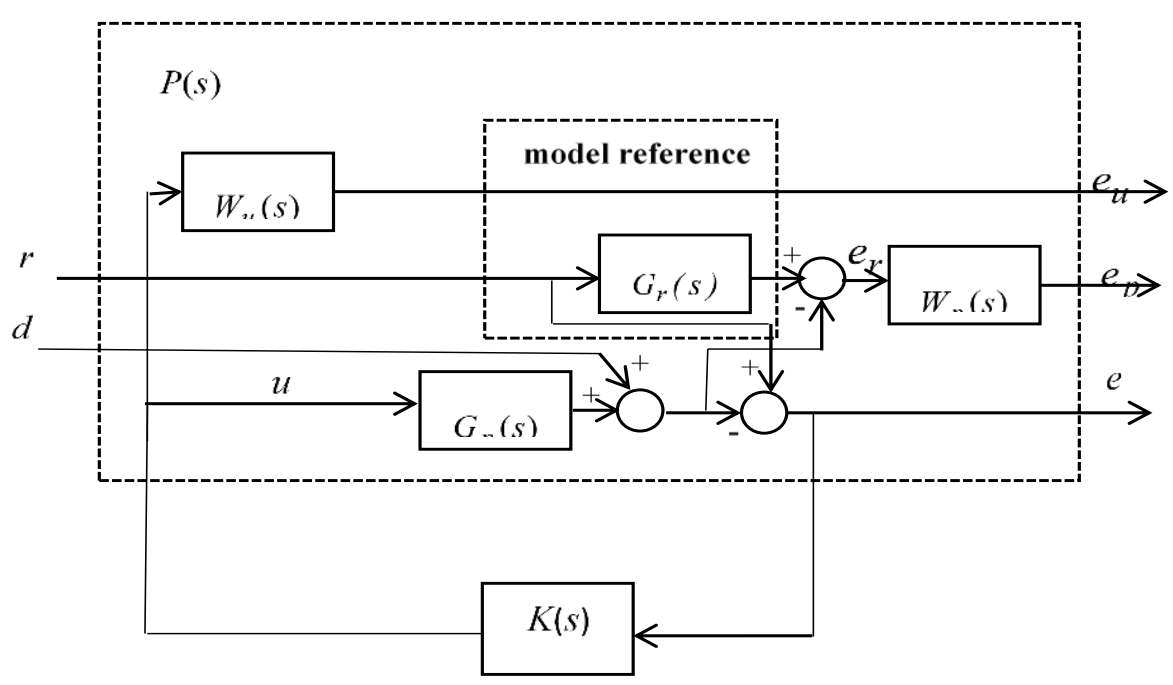

Figure 6: The overall block diagram of the system with model reference

where

$$
F_{l}(P, K)=\left[P_{11}(s)+P_{12}(s) K(s)\left\{I-P_{22}(s) K(s)\right\}^{-1} P_{21}(s)\right]
$$

The generalized plant $P$ can be expressed as:

$$
\left[\begin{array}{l}
e_{p} \\
e_{u} \\
e
\end{array}\right]=P\left[\begin{array}{l}
r \\
d \\
u
\end{array}\right]=
$$

$$
\begin{aligned}
& \text { model } \\
& \text { reference } \\
& {\left[\begin{array}{ccc}
G_{r} W_{p} & -W_{p} & -G_{p} W_{p} \\
\hdashline-\cdots & 0 & W_{u} \\
1 & -1 & -G_{p}
\end{array}\right]\left[\begin{array}{l}
r_{1} \\
d \\
u
\end{array}\right]}
\end{aligned}
$$

then

$$
\text { model }
$$

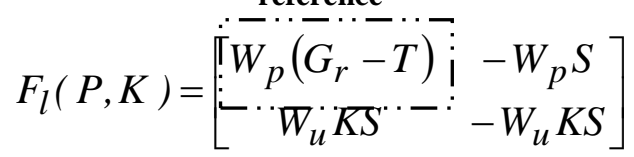

where $S(s)=\frac{1}{1+G_{p}(s) K(s)}$ represents sensitivity function and $T(s)=\frac{G_{p}(s) K(s)}{1+G_{p}(s) K(s)} \quad$ represents complementary sensitivity function respectively.

The H-infinity optimal control synthesis procedure consists of finding a controller $K(s)$ that stabilizes the system such that the infinite norm of the closed loop transfer matrix $F_{l}(P, K)$ is minimized, that is [11]:

$$
\left\|F_{l}(P, K)\right\|_{\infty} \leq \gamma
$$

where $\|.\|_{\infty}$ represents the $\mathrm{H}_{\infty}$ norm of $F_{l}(P, K)$, and $\gamma$ is a positive number.

To add the uncertainty model to the system, the Lower Fractional Transformation (LFT) is transformed to the Upper Fractional Transformation (UFT) by obtaining $N$ which is equal to $F_{l}(P, K) w$ [11]. On the other hand, both structured and unstructured uncertainties are considered in $\mathrm{H}$ infinity control design. However, for more control over the system, the unstructured uncertainty can be used to cover the high frequencies unmodeled system dynamics. The uncertainty model is added to the system as shown in Figure 7 to form the Upper Fractional Transformation (UFT). The overall block diagram of the system with multiplicative uncertainty and the added model reference is shown in Figure 8 . Where $W_{m}$ represents the multiplicative uncertainty model of the system. The overall system can be represented by:

$$
\begin{aligned}
& {\left[\begin{array}{c}
y \Delta \\
z_{1} \\
z_{2} \\
v
\end{array}\right]=P\left[\begin{array}{c}
u \Delta \\
r \\
d \\
u
\end{array}\right]=\begin{array}{c}
\text { model } \\
\text { reference }
\end{array}} \\
& {\left[\begin{array}{ccccc}
0 & 0 & 0 & W_{m} G_{p} \\
-W_{p} & \vdots W_{p} G_{r} & -W_{p} & -W_{p} G_{p} \\
0 & -\cdots & 0 & W_{u} \\
-1 & 1 & -1 & -G_{p}
\end{array}\right]\left[\begin{array}{c}
u \Delta \\
r \\
d \\
u
\end{array}\right]}
\end{aligned}
$$




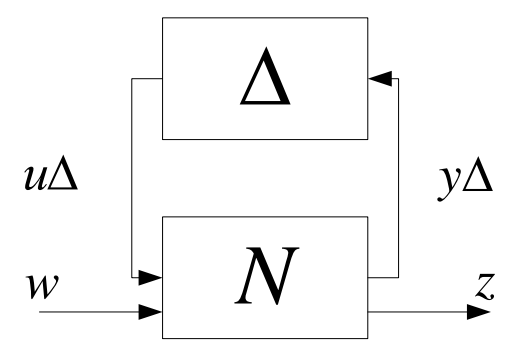

Figure 7: Upper fractional transformation (UFT)

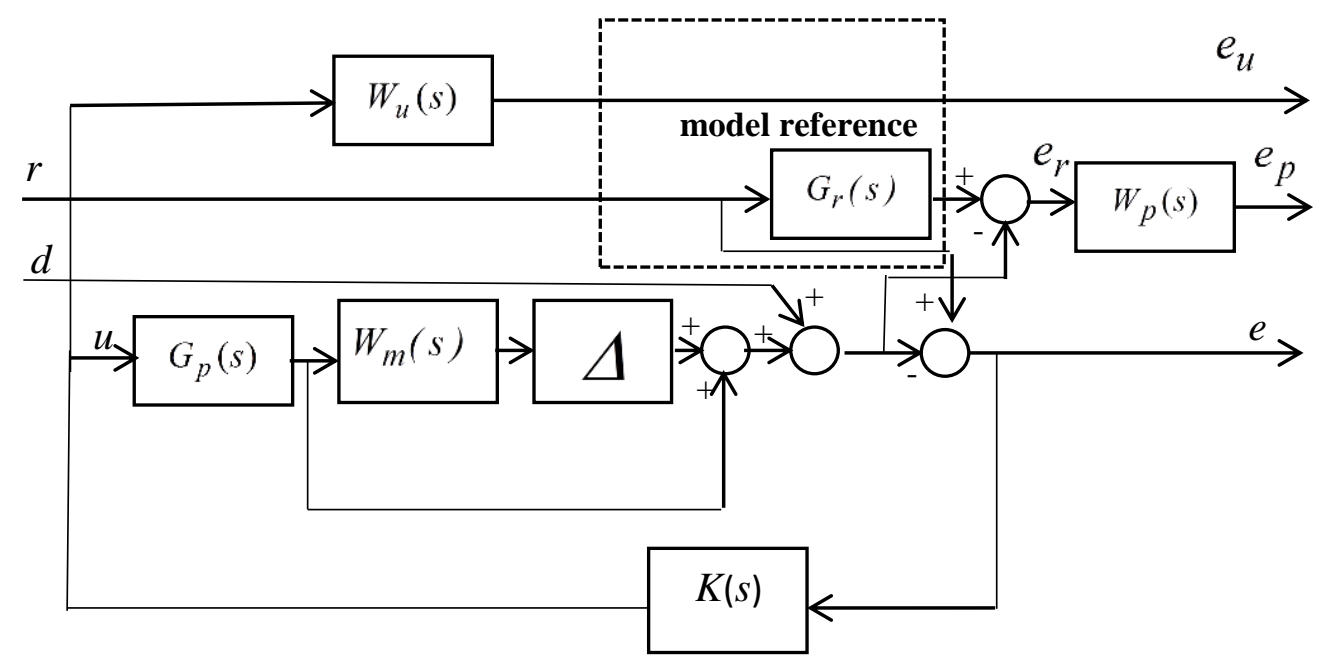

Figure 8: Block diagram of the system with model reference and weights

The augmented plant $P$ and the controller $K$ can be represented by LFT as: $F_{l}(P, K)=N=\left[P_{11}(s)+P_{12}(s) K(s)\left\{I-P_{22}(s) K(s)\right\}^{-1} P_{21}(s)\right]$ (17) and,

$$
\text { model }
$$
reference

$N=\left[\begin{array}{ccc}-W_{m} T & \searrow \cdot W_{m} T & -W_{m} T \\ -W_{p} S & \vdots W_{p}\left(G_{r}-T\right) \vdots & -W_{p} S \\ -W_{u} K S & -\cdot \bar{W}_{u} \cdot \overline{K S} \cdot & -W_{u} K S\end{array}\right]$

The transfer function with uncertainty that relates $w$ to $z$ can be found using Upper Fractional Transformational (UFT) function as [11]:

$$
z=F_{u}(N, \Delta) w=\left[N_{22}+N_{21} \Delta\left(1-N_{11} \Delta\right)^{-1} N_{12}\right] w
$$

The H-infinity control objective is to obtain the controller $K(s)$ which guarantees the internal stability of the system by minimizing $F_{u}(N, \Delta)$. The minimization procedure depends on $\gamma$. iteration in obtaining the stabilizing controller such that:

$\left\|F_{u}(N, \Delta)\right\|_{\infty}<\gamma$
It is shown that the cost function to be minimized is a function of $(N)$ which is in turn a function of the error between the two outputs (system and model reference). The controller parameters will be adjusted in such away so that the cost function is minimized. The performance and control weighting functions are set manually by trial and error to meet the required robust stability and performance. The selected weighting functions are:

$$
\begin{aligned}
& W_{p}(s)=\frac{5}{0.1 s+0.001} \\
& W_{u}(s)=10^{-5}
\end{aligned}
$$

The multiplicative uncertainty weighting function is calculated to cover $\pm 10 \%$ variation in system parameters $(R, L$ and $m)$. The obtained uncertainty weight is:

$W_{m}(s)=\frac{0.1774 s+8.717}{s+39.03}$

Figure 9 shows the proposed block diagram of $\mathrm{H}$ infinity model reference. The model reference transfer function is selected as a third order by considering ITAE criterion (integral time absolute error) which is related to the desired settling time. The considered model reference structure is [13, 14]:

$$
G_{r}(s)=\frac{w_{n}^{3}}{s^{3}+1.75 w_{n} s^{2}+2.15 w_{n}^{2} s+w_{n}^{3}}
$$


where $w_{n}$ represents the model reference natural frequency and its value is selected to be $10^{5}$ rad/sec. which satisfies $25 \mathrm{msec}$. settling time.

\section{Results and Discussion}

Figure 10 shows the closed loop time response of the system. It is clear that the system is unstable. To stabilize the system, the proposed H-infinity controller is applied. The obtained controller is:

$K(s)=$

$-2.085 \times 10^{18} s^{3}-1.486 \times 10^{20} s^{2}-3.484 \times 10^{21} s-2.684 \times 10^{22}$

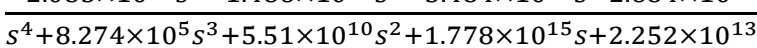
(25)

Figure 11 shows the frequency response for complementary sensitivity function $(T)$ and sensitivity function $(S)$. Figures 12 shows the frequency response for sensitivity function $(S)$ and performance weighting function $\left(W_{p}^{-1}\right)$ and the frequency response of complementary sensitivity function $(T)$ and uncertainty weighting function $\left(W_{m}^{-1}\right)$ is shown in Figure 13. From these figures it is shown that the magnitudes of $(S)$ and $(T)$ are less than the magnitudes of $\left(W_{p}^{-1}\right)$ and $\left(W_{m}^{-1}\right)$ respectively and for all frequencies which means that the robust performance and robust stability conditions have been achieved. The characteristics of the control signal are shown in Figure 14. It is shown that the magnitudes of $(K S)$ are less than the magnitudes of the inverse of the control weighting function $\left(W_{u}^{-1}\right)$. The time response specifications of the controlled system using $\mathrm{H}$-infinity and $\mathrm{H}$ infinity model reference controllers are shown in Figures 15 and 16 respectively.

It is clear that the output of the system using the proposed $\mathrm{H}$-infinity model reference controller has closely tracked the desired specifications represented by the output of the given model reference. Further, it is shown that the proposed controller can achieve a robust performance better than if only one of them is used. The time response of the controlled uncertain system is shown in Figure 17. A variation of $\pm 10 \%$ in system parameters $(R, L$ and $m)$ was considered. It shows that the system stability has been guaranteed with the variations in system parameters with achieving the desired tracking represented by the selected model reference.

On the other hand, as compared to the standard $\mathrm{H}$ infinity control, the proposed controller is very effective in obtaining an adequate transient response represented by the model reference which its output has been used as a desired system output response.

\section{Conclusion}

In this paper the model reference control has been combined with $\mathrm{H}$-infinity approach to obtain a new robust control for the magnetic levitation system. In this design, the controller parameters have been adjusted such that the system output tracks the output of the model reference. The proposed controller has achieved an asymptotic tracking of prescribed reference output with compensating the system parameters uncertainty. A variation of $\pm 10 \%$ in system parameters has been considered. Finally, it was shown that the proposed controller could overcome the disadvantage of the H-infinity controller in obtaining adequate time response specifications.

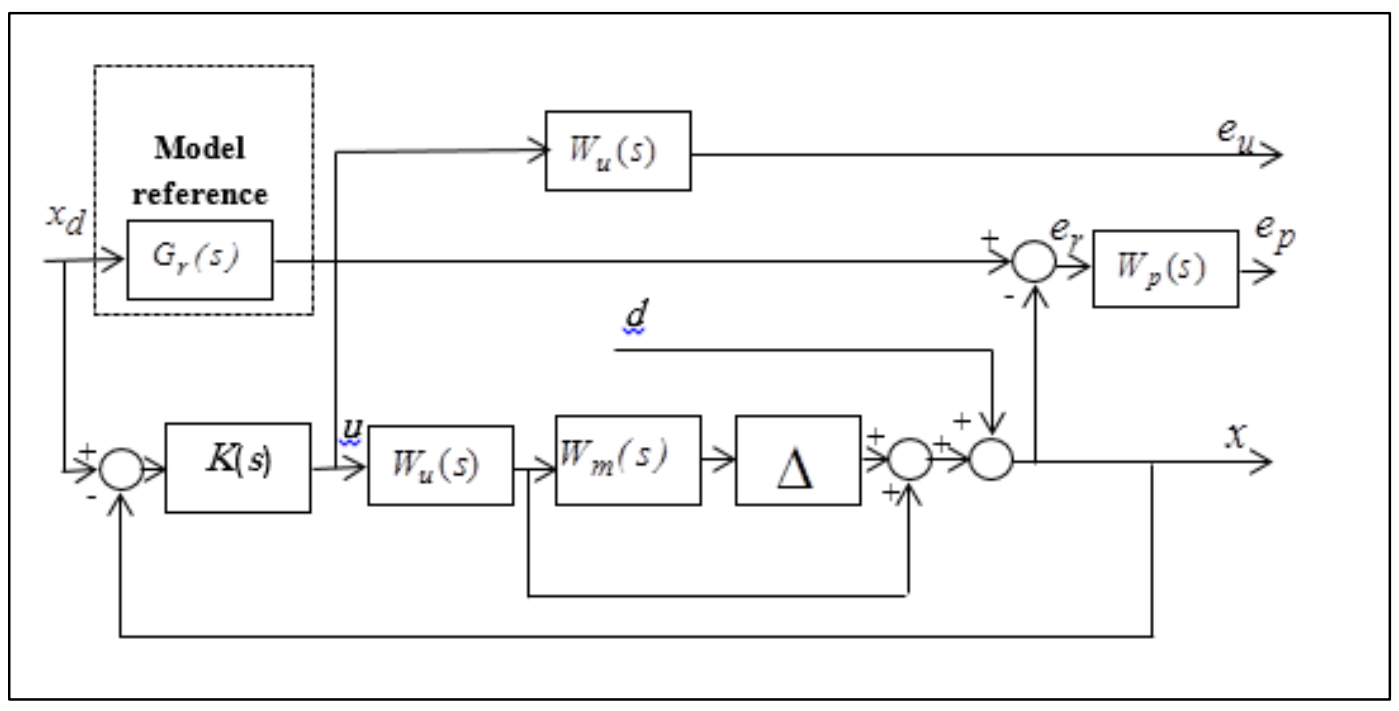

Figure 9: The entire system block diagram with model reference and weights 


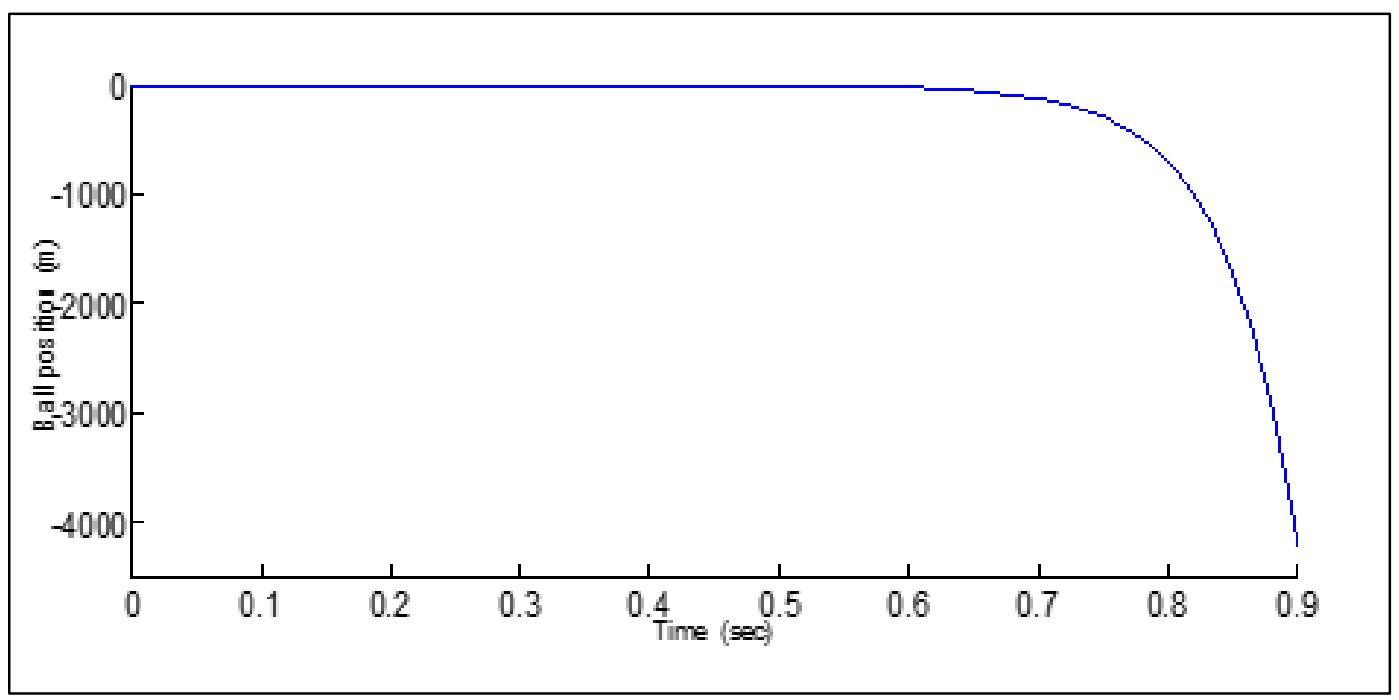

Figure 10: Time response specifications of the system before applying the proposed controller

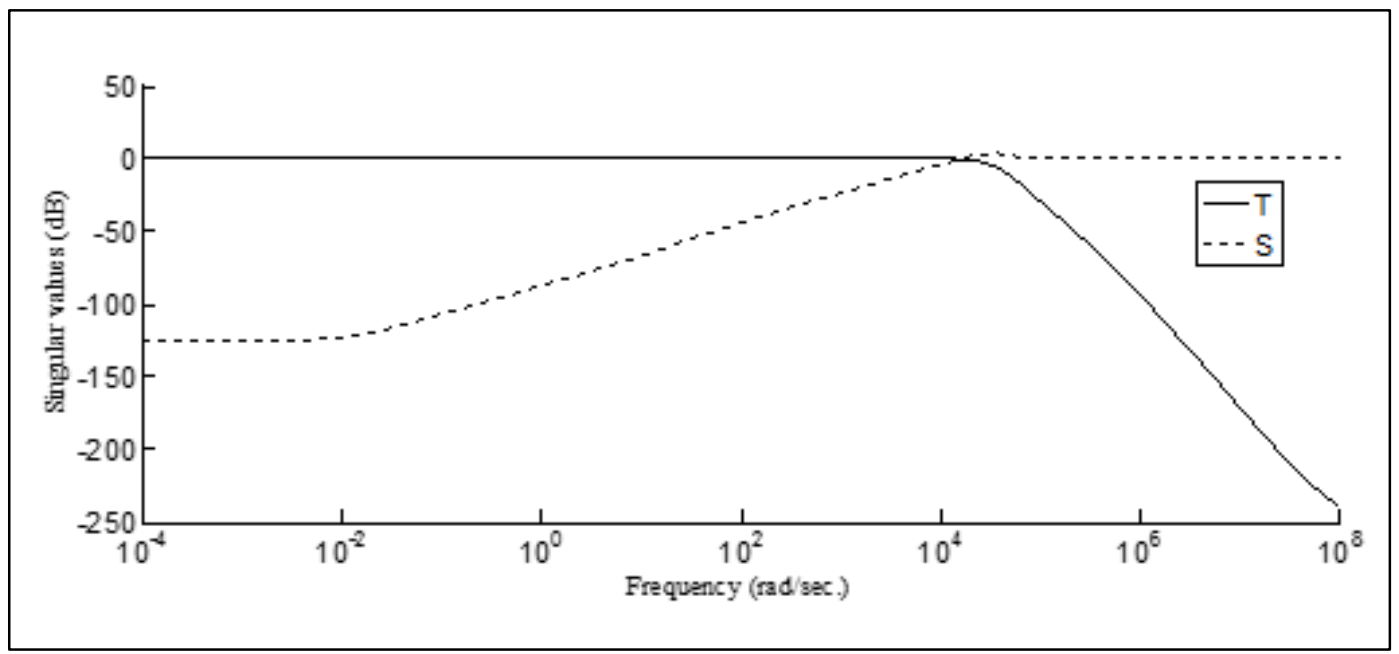

Figure 11: Frequency response characteristics of the complementary sensitivity function (T) and sensitivity function (S)

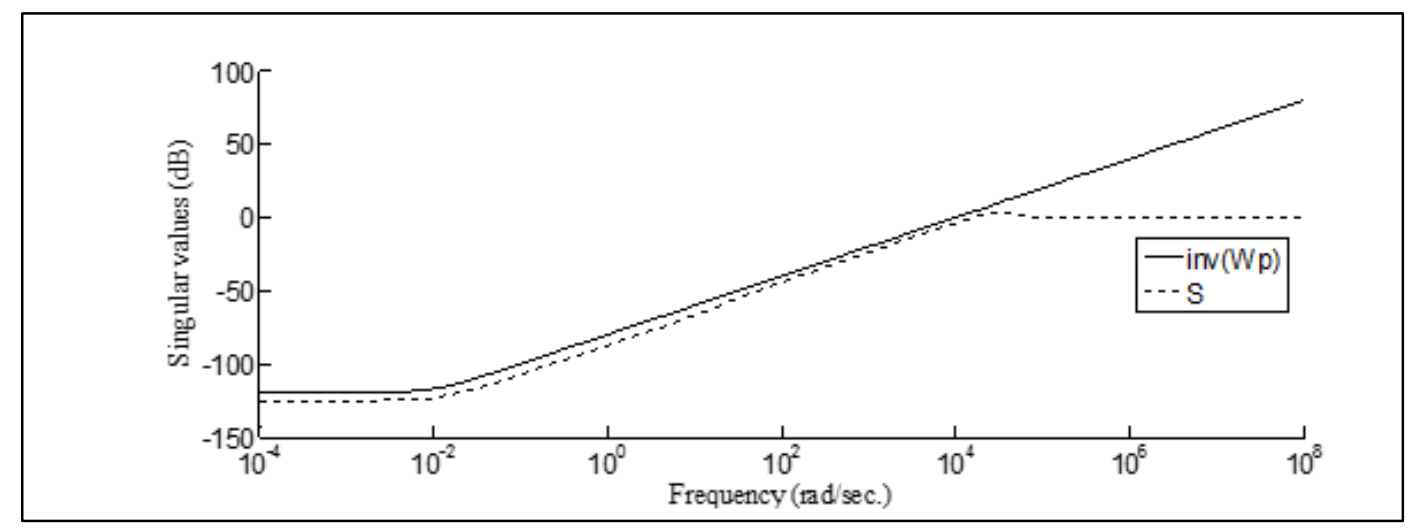

Figure 12: Frequency response of the sensitivity function $(S)$ and $\left(W_{p}^{-1}\right)$ 


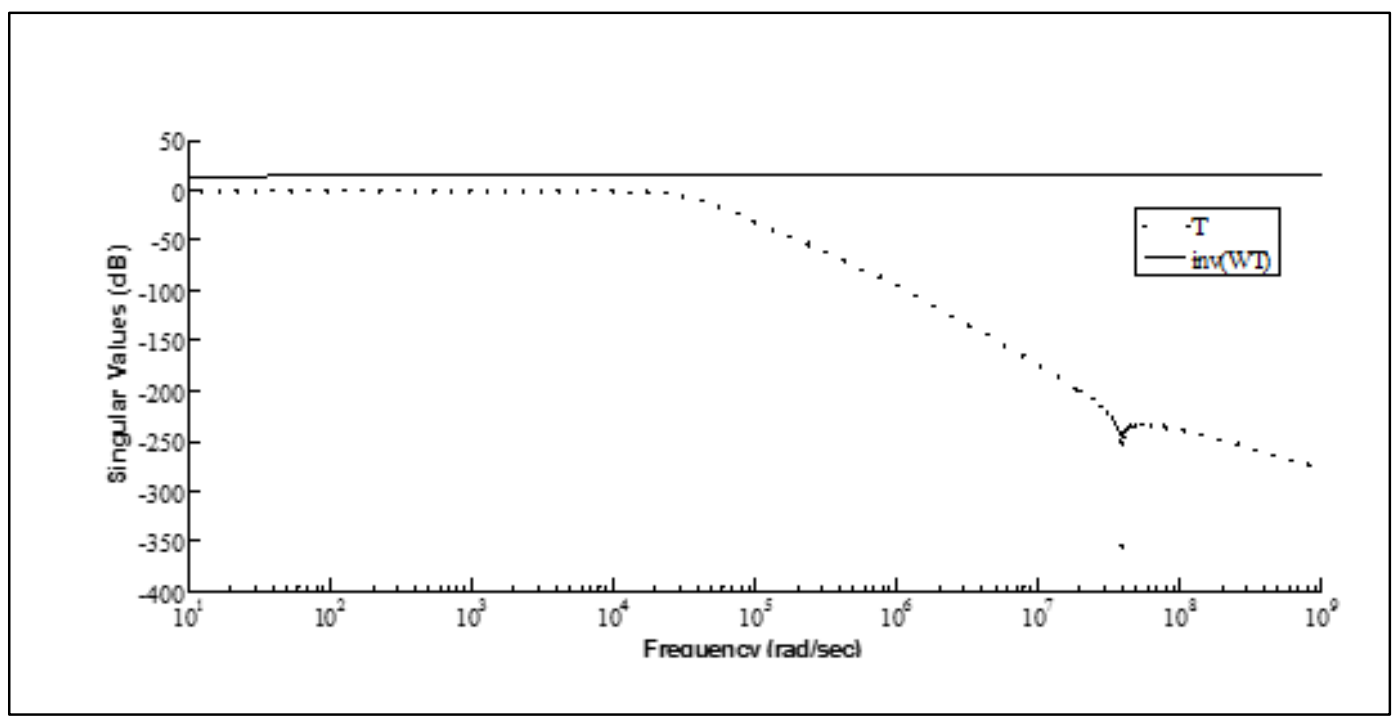

Figure 13: Frequency response of the complementary sensitivity function $(\mathrm{T})$ and $\left(W_{m}^{-1}\right)$

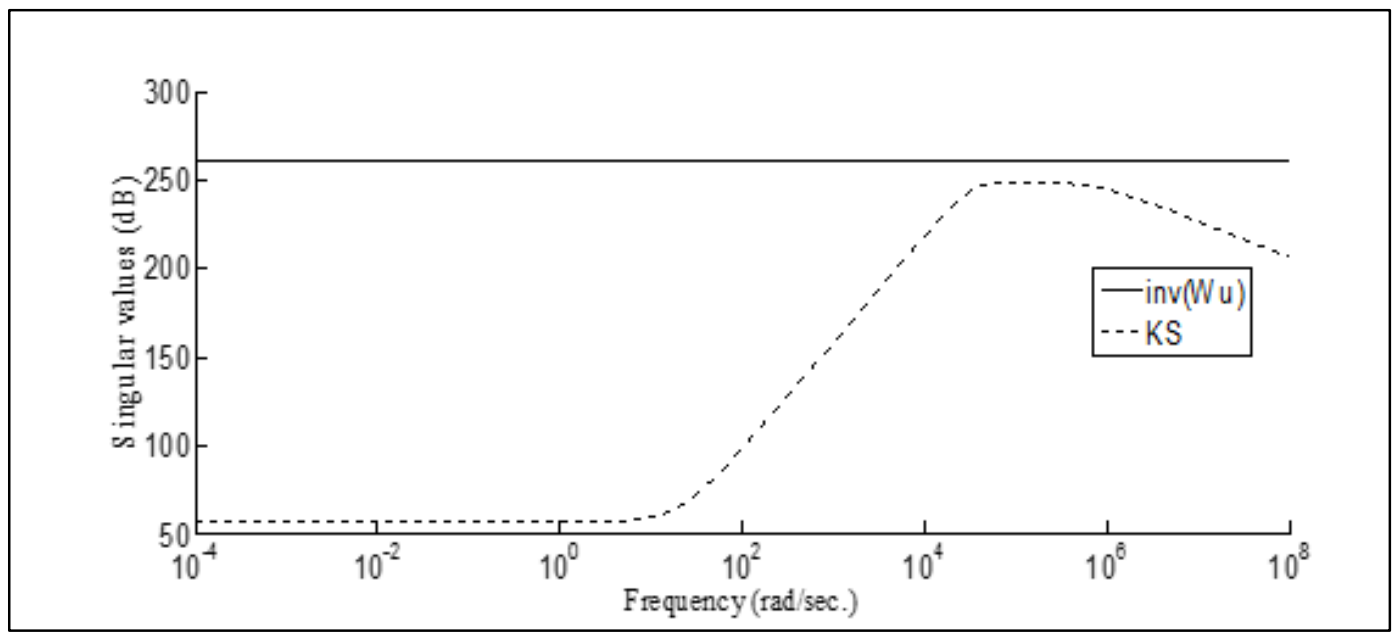

Figure 14: Frequency response of the control sensitivity function (KS) and $\left(W_{u}^{-1}\right)$

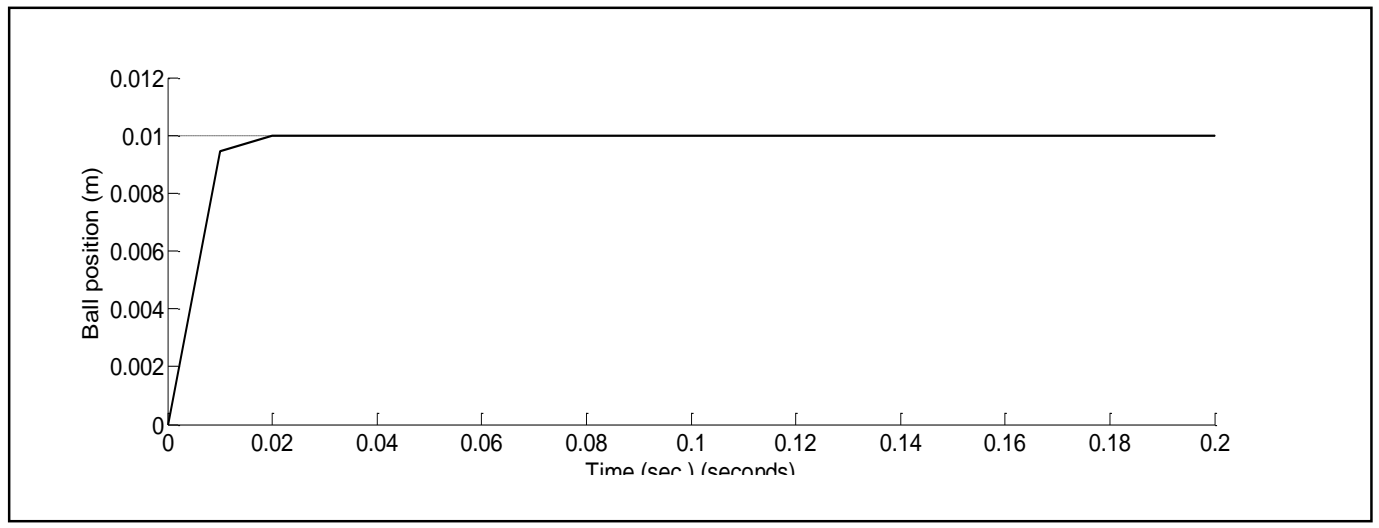

Figure 15: Time response specifications of the controlled system using $\mathrm{H}$-infinity controller 


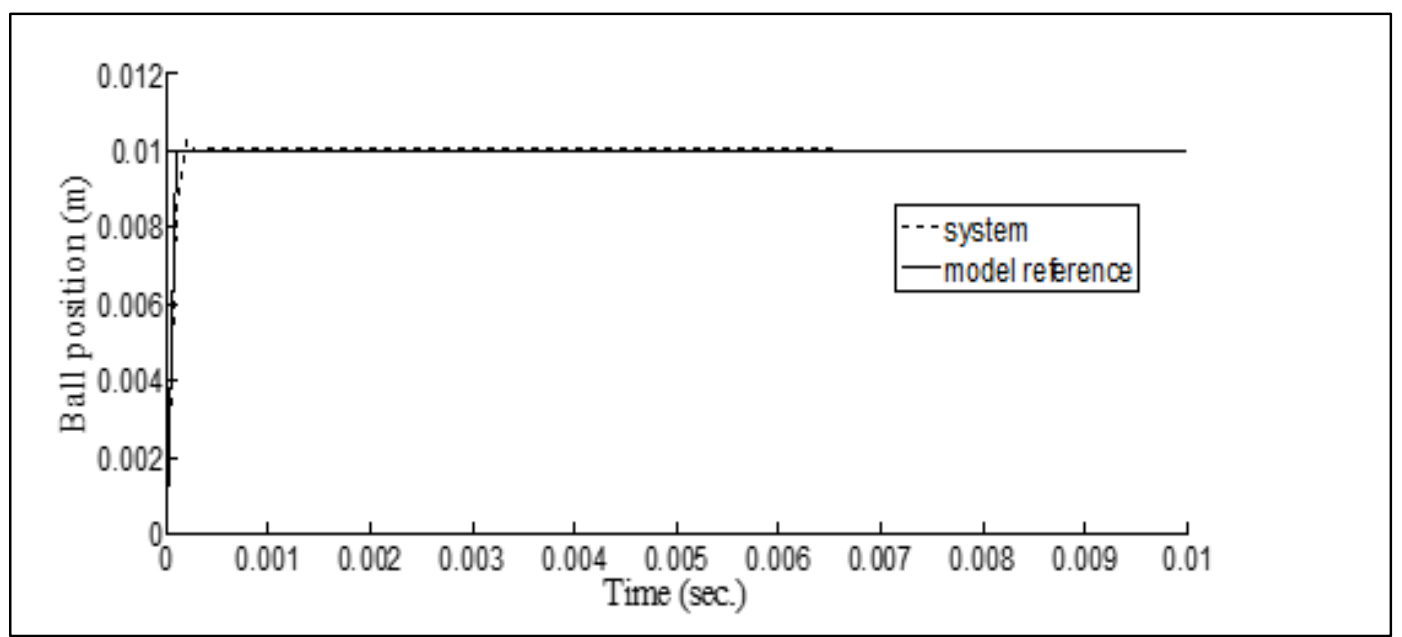

Figure 16: Time response specifications of the controlled system using $\mathbf{H}$-infinity model reference Controller

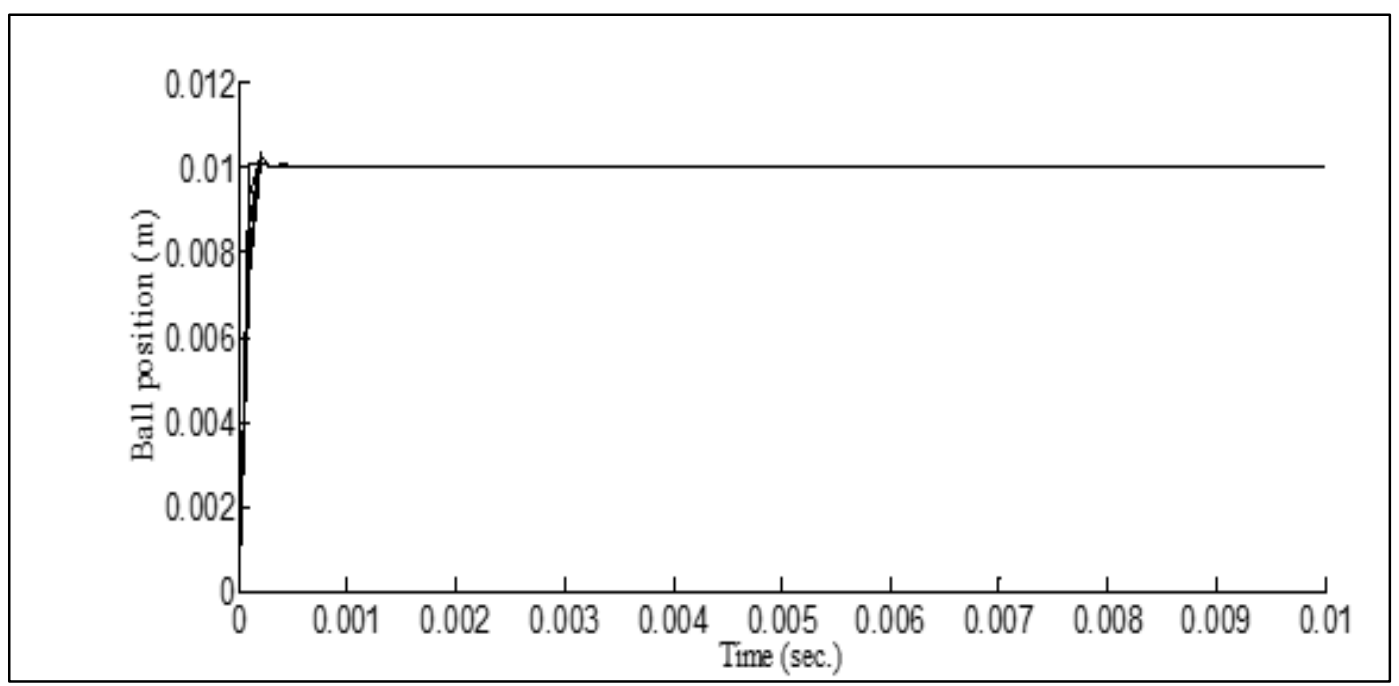

Figure 17: Time response specifications of the controlled uncertain system

\section{References}

[1] S.K. Choudhary, "Robust Feedback Control Analysis of Magnetic Levitation System”, WSEAS Transactions on Systems. Vol. 13, pp. 285-291, 2014.

[2] I. Ahmad and M. A. Javaid, "Nonlinear Model and Controller Design for Magnetic Levitation System", Recent Advances in Signal Processing, Robotics and Automation, University of Engineering \& Technology, Taxila, Pakistan, 2012.

[3] A. Kumar, M.K. Panda, S. Kundu and V. Kumar, "Designing of an Interval Type-2 Fuzzy Logic Controller for Magnetic Levitation System with Reduced Rule Base", IEEE, 12th International Conference on Computing, Communications and Networking Technologies, India, 2012.

[4] H.K. Chiang, C.C. Fang and G.W. Chen, "Second Order Siding Mode Control with Fuzzy Compensator for a Magnetic Levitation System", IEEE, Second International Conference on Digital Manufacturing and Automation, Hunan, China, 2011.

[5] Z.J. Yang, Y. Fukushima, S. Kanae and K. Wada, "Robust Non-Linear Output Feedback Control of a Magnetic Levitation System by K-Filter Approach”, IET
Control Theory and Applications, Vol. 3, No. 7, pp. 852-864, 2009.

[6] H.I. Ali, "Robust PI-PD Controller Design for Magnetic Levitation System", Engineering and Technology Journal, Vol. 32, Part (A), No. 3, pp. 667 680, 2014.

[7] S.J. Cheng, "Hळ Control and Sliding Mode Control of Magnetic Levitation System", Asian Journal of Control. Vol. 4, No. 3, pp. 333-340, 2002.

[8] G. Suganya, L. J. Amla and S. P. Dwarakesh, "Model Reference Adaptive Controller using MOPSO for a Nonlinear Boiler Turbine", Vol. 4, No. 4, pp. 87-91, 2014.

[9] "Magnetic Levitation Control 33-942 S", Feedback Instruments team Ltd., UK, 2009.

[10] H.I. Ali and M. I. Abd, "Optimal Multi-objective Robust Controller Design for Magnetic Levitation System, IJCCCE, Vol. 15, No. 1, pp. 18-34, 2015.

[11] Z. Kemin and D. Jhon, "Essentials of Robust Control", Upper Saddle River, New Jersey: Prentice Hall Inc., 1998.

[12] S. Alok, "Linear Systems Optimal and Robust Control”, Taylor and Francis Group, USA, 2007. 
[13] P. Jain and M. J. Nigam, "Design of a Model Reference Adaptive Controller using Modified MIT Rule for a Second Order System", Vol. 3, No. 4, pp. 477-484, 2013.

[14] A. A. Aly, "Model Reference PID Control of an Electro-hydraulic Drive", International Journal of Intelligent Systems and Applications", Vol. 11, pp. 2432, 2012.

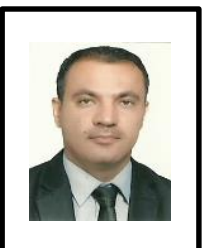

\section{H. I. Ali}

He graduated from the department of Control and Systems Engineering, University of Technology, Baghdad, Iraq in 1997. He obtained the MSc degree in Mechatronics Engineering from University of Technology, Baghdad, Iraq in 2000 and the $\mathrm{PhD}$ in Control and Automation from the Department of Electrical and Electronics Engineering, University Putra Malaysia, Malaysia in 2010. Currently he is an Assistant Professor in Control and Systems Engineering Department, University of Technology, Baghdad, Iraq. His current research interests include robust control, intelligent control and optimization techniques. He is a member of IEEE and IAENG. 